\title{
Enhancement of Probe Density in DNA Sensing by Tuning the Exponential Growth Regime of Polyelectrolyte Multilayers
}

\author{
Jacopo Movilli, Salmeen Shakil Choudhury, Monika Schönhoff,* and Jurriaan Huskens*
}

Cite This: Chem. Mater. 2020, 32, 9155-9166

Read Online

ABSTRACT: Surface-based biosensing devices benefit from a dedicated design of the probe layer present at the transducing interface. The layer architecture, its physicochemical properties, and the embedding of the receptor sites affect the probability of binding the analyte. Here, the enhancement of the probe density at the sensing interface by tuning the exponential growth regime of polyelectrolyte multilayers (PEMs) is presented. PEMs were made of poly-L-lysine (PLL), with appended clickable dibenzocyclooctyne (DBCO) groups and oligo(ethylene glycol) chains, and poly(styrene sulfonate) (PSS). The DNA probe loading and target hybridization efficiencies of the PEMs were evaluated as a function of the PLL layer number and the growth regime by a quartz crystal

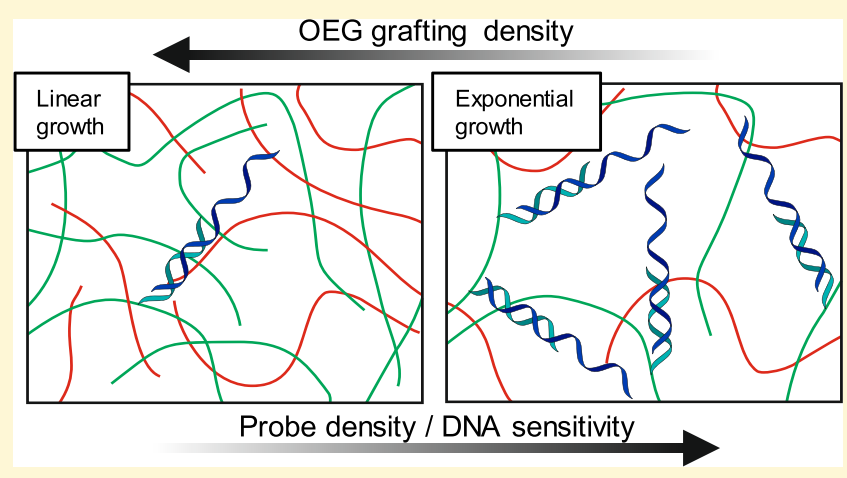
microbalance (QCM). An amplification factor of 25 in the target

DNA detection was found for a 33-layer exponentially grown PEM compared to a monolayer. A Voigt-based model showed that DNA probe binding to the DBCO groups is more efficient in the open, exponentially grown films, while the hybridization efficiencies appeared to be high for all layer architectures. These results show the potential of such engineered gel-like structures to increase the detection of bio-relevant analytes in biosensing systems.

\section{INTRODUCTION}

The possibility of detecting and monitoring biologically relevant molecules has changed the way of approaching scientific challenges in biology, ${ }^{1,2}$ medicine, ${ }^{3}$ and food and environmental control. ${ }^{4}$ Among others, DNA biosensors have attracted the general interest of the scientific community due to the potential implications in the life sciences $^{5-8}$ as well as future data storage technology. ${ }^{9}$ The possibility of specifically recognizing target DNA sequences has enhanced the capability of performing reliable diagnostics for disorders associated with the variation in regulatory $\mathrm{DNA}^{10}$ such as neurodegenerative diseases, ${ }^{11}$ cystic fibrosis, ${ }^{12}$ and cancer. ${ }^{13}$

Free circulating DNA molecules can act as biomarkers. ${ }^{14,15}$ The possibility of monitoring the concentrations of such species directly from body fluid samples (liquid biopsy), in contrast to the costly and tedious traditional method (tissue biopsy), has fueled the development of detection technologies to improve the prevention, diagnosis, and monitoring of genetic diseases. ${ }^{16}$ However, despite recent progress, specifically for the more practical surface-based DNA biosensors, ${ }^{17,18}$ the low concentration of DNA biomarkers in body fluids ${ }^{15}$ has held back the wide applicability of such devices. Therefore, signal amplification strategies are commonly exploited with DNA sensing platforms to achieve a lower limit of detection (LOD). ${ }^{19-21}$

The efficiency of DNA hybridization, as well as any subsequent signal enhancement strategy, is affected by the physicochemical characteristics of the sensing interface. $^{22,23}$ Controlling the formation of molecular assemblies with defined composition and structure plays a fundamental role in the creation of functional DNA sensing devices with excellent selectivity and sensitivity. ${ }^{24-26}$ The surface presentation of the anchored probes defines the surface probe density and the overall hybridization process. ${ }^{27,28}$ Moreover, antifouling moieties are often required to minimize non-specific interactions at the surface, consequently increasing the signal-to-noise ratio during the signal enhancement. ${ }^{29,30}$

The layer-by-layer (LbL) assembly approach, which is based on the alternating deposition of oppositely charged species, typically polyelectrolytes (PEs), ${ }^{31}$ has been extensively used to tailor surface layers with micro and nanostructured features at interfaces for biomedical applications. ${ }^{32}$ Polyelectrolyte multilayers (PEMs) have shown to provide distinct structural architectures based on the type of growth regime (defined as the growth mechanism of the PEM, exponential or linear) due to the diffusion of partially ionized ("weak") PEs inside the

Received: June 11, 2020

Revised: October 12, 2020

Published: October 26, 2020 


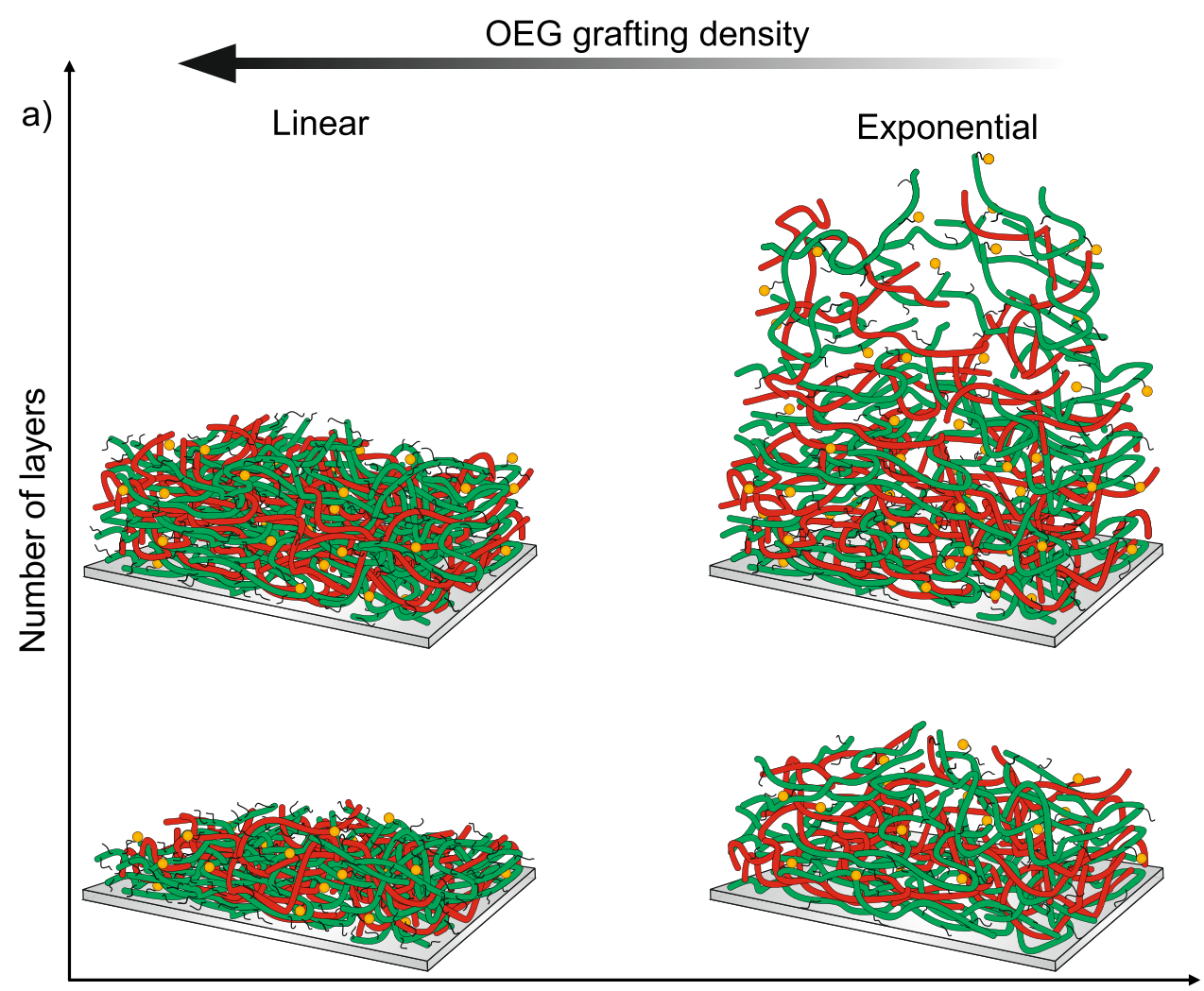

Density of unmodified lysine moieties

b)

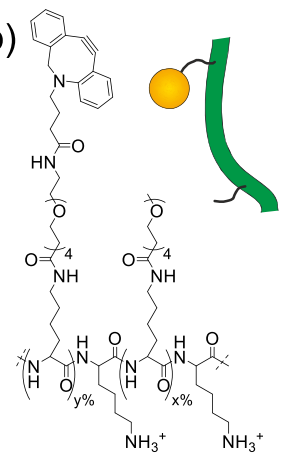

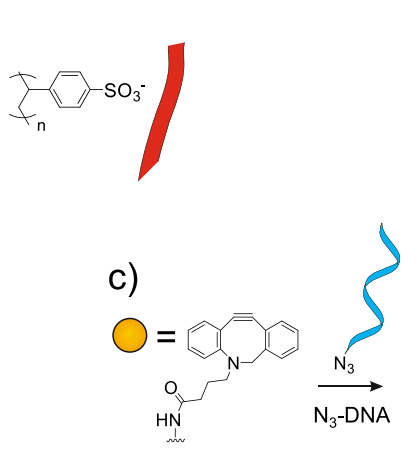

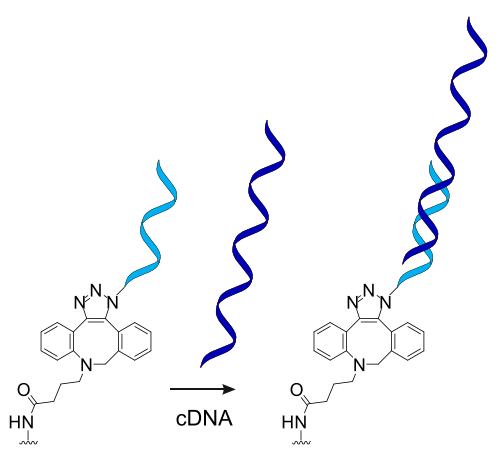

Figure 1. (a) Illustration showing the control over the mechanism of the $\mathrm{PE}$ multilayer formation and its final thickness on activated $\mathrm{SiO}_{2}$ substrates by varying the grafting density of OEG on the PLL backbone and the number of PE layers. The PEMs were formed by alternating deposition of PSS (red) and modified PLL (green), which provides the relative density of both OEG (black thin line) and DBCO (yellow sphere) moieties within the architectures. (b) Structures of the PLL-OEG-DBCO and PSS polyelectrolytes used. (c) Schematic representation displaying the chemistry of the process for anchoring DNA-azide to the PEMs via SPAAC click chemistry followed by a cDNA recognition step. All DNA sequences used in this work are reported in Table S1. The structure of the azide linker of the DNA probe is shown in Figure S1.

PEM. $^{33,34}$ By varying chemical characteristics and assembly conditions of the constituent $\mathrm{PEs},{ }^{35-37}$ softer and more hydrated (gel-like) structures can be formed, ${ }^{38}$ ensuring additional control over the mobility and "openness" within the architecture. ${ }^{39,40}$ Diverse LbL structures have been used for DNA biosensors due to the appealing property of providing a high loading of molecules onto a surface. ${ }^{41}$ However, these documented LbL-based DNA biosensors have DNA probes either as a constituent layer (or layers) ${ }^{42}$ or randomly anchored to linker moieties physisorbed on PEMs, such as avidin or dendrimers. ${ }^{43-45}$ Such LbL assemblies provide highly dense DNA films without tuning of the growth regime (which is exclusively linear) or the probe density, thus providing little control over the hybridization efficiency and the sensitivity itself. $^{46}$
Here, we show the use of tailored PEM architectures with appended antifouling and clickable groups for controlled and enhanced DNA loading and sensitivity. We envisage the creation of extended probe binding capacity by tuning the PEM formation into the exponential growth regime, which provides extended surface coverage and, similarly beneficial, a more open architecture. In order to tune the poly-L-lysine/ poly(styrene sulfonate) (PLL/PSS) multilayer growth regime, we varied the grafting ratio of oligo(ethylene glycol) units (OEG) appended to PLL (PLL-OEG) while at the same time maintaining the fluidity and the antifouling behavior. ${ }^{47-49}$ PLL-OEG polymers with appended bio-orthogonal dibenzocyclooctyne (DBCO) moieties (PLL-OEG-DBCO) were exploited to ensure the control of the DNA probe density $^{25,50,51}$ within the multilayer system for better 
evaluation of the DNA hybridization efficiency. An azido-DNA probe with a sequence complementary to the KRAS gene, mutations of which are associated with, among others, several types of cancers, ${ }^{52}$ was anchored to the DBCO-functionalized PEM structures. This way, the final biorecognition architectures were prepared, with the potential application for tumor DNA detection. Samples expressing the same growth regime but having different numbers of layers and samples with equal layer numbers but made under different growth regimes were compared. Their capacity to bind probe DNA molecules and to recognize complementary DNA (cDNA) sequences was evaluated by recording the frequency shifts of each step by means of a quartz crystal microbalance with dissipation monitoring (QCM-D). A viscoelastic Voigt-based model was exploited to assess the effective thickness of the multilayer film structures and to compare the content of DBCO groups within the PEMs with the amount of detected DNA for evaluating the effects of the multilayer film properties on the amplified sensitivity. Additionally, the evaluation of the probe binding capacity and the comparison of the dry and wet thicknesses of the multilayers are used to assess the open character of the exponentially grown multilayers.

\section{RESULTS AND DISCUSSION}

Concept of Growth Regime Control and Enhanced DNA Sensitivity. Figure 1a shows the concept of controlling the growth regime and the PEM architecture by the targeted design of the modified PLL. By tuning the percentage of grafted OEG groups, the openness of the multilayer architecture is affected, and enhanced DNA detection performance and sensitivity are achieved. The molecule accessibility is dictated by the type of growth, related to the internal structure of the final architecture, and ultimately to the diffusion of the "weak" PLL within the PEM. 35,39 The formation of the multilayer films was achieved on $\mathrm{SiO}_{2}$ QCM-D substrates by the alternating deposition of the polyelectrolytes PLL-OEG-DBCO and PSS (green and red lines in Figure 1a,b, respectively). Increasing numbers of layers result in higher thicknesses of the multilayers owing to the larger adsorbed mass ( $y$-axis). On the other hand, the OEG mol fraction affects the growth mechanism of the PEM itself ( $x$-axis). The "weak" polyelectrolyte nature of PLL, ${ }^{53}$ in combination with the "strong" PSS, provides the "in" and "out" diffusion process resulting in an exponential growth regime (Figure la right). ${ }^{54}$ As we will show (see below), varying the OEG content bestows a way to control the growth law (Figure la left), providing suppressed exponential growth, even reaching (pseudo-)linear growth and completely suppressed growth. In addition, the projected DBCO density increases with the total number of layers, regardless of the type of growth mode (Figure 1a, top versus bottom). The biorecognition capability of the PEM architectures was investigated by reacting the PLL-OEG-DBCO with a DNAazide probe $(23 \mathrm{nt})$ by strain-promoted azide-alkyne cycloaddition (SPAAC) click chemistry ${ }^{50,55}$ followed by hybridization with cDNA (43 nt) (Figure 1c). As a proof of principle for the improved sensitivity, the QCM responses to the DNA probe and cDNA were evaluated in relation with the number of layers present in the multilayer structure.

Effect of OEG and DBCO Appended Groups on the LbL Growth Regime. The modified PLL was synthesized by following procedures reported previously. ${ }^{25,50}$ PLL polymers $(15-30 \mathrm{kDa})$ were grafted with spacers having four oligo- (ethylene glycol) units terminated with either methoxy (OEG) or DBCO units. The percentage of lysine monomers functionalized with the OEG group ranged between 0 and $35 \%$, while the DBCO grafting density was kept constant at approx. $2 \%$. Besides the main function of controlling the growth mechanism of the multilayer, the OEG moieties also minimize the non-specific interactions, ${ }^{49,56}$ in concert with the zwitterionic-like nature of the PEM film. ${ }^{57,58}$ Moreover, the OEG grafting density is crucial to control the fluidity of the structure by affecting the diffusivity of the PLL within the PEM, as was shown to occur for the weak PEs pegylated poly(ethylenimine) and hyaluronic acid. ${ }^{48}$ The grafting densities of OEG and DBCO appended to the PLL backbone have been determined by ${ }^{1} \mathrm{H}$ NMR according to a previously reported method (Table S2 and an NMR example of modified PLL in Figure S2). ${ }^{25,50}$

In order to study the influence of the OEG grafting density on the type of growth regime, modified PLLs grafted with varying OEG mol fractions were tested in LbL assembly. PLLOEGs $(0.5 \mathrm{mg} / \mathrm{mL}$ solutions) were adsorbed onto freshly oxygen plasma-activated $\mathrm{SiO}_{2}$ QCM substrates followed by PSS deposition $(0.5 \mathrm{mg} / \mathrm{mL})$. Although a procedure to achieve exponential growth of multilayers of unfunctionalized PLL and PSS has been reported using aqueous $\mathrm{NaCl}$ solution (150 $\mathrm{mM}),{ }^{35,59}$ we here employed PBS buffer at $\mathrm{pH} 6.4$ with the same ionic strength. The buffer solution was exploited to minimize local apparent $\mathrm{p} K_{\mathrm{a}}$ fluctuations of PLL that occur upon adsorption, which could lead to undesired secondary conformational changes within the $\mathrm{PEM}^{53}$ and possibly to a shift between growth regimes. ${ }^{60}$ In this system with PLL and PSS, the weak PE PLL is expected to be the only polyelectrolyte diffusing to cause the exponential growth. ${ }^{54,61,62}$ The PLL diffusion process induces the exponential growth behavior of the PEM architecture, ${ }^{63}$ which is most pronounced between $\mathrm{pH} 5.0$ and 7.0 for PEMs formed with PLL/hyaluronic acid, as observed by Bütergerds and coworkers. ${ }^{62}$ Therefore, PBS at $\mathrm{pH} 6.4$ is a good compromise to fulfill the prerequisites mentioned above, and it was used for all the experiments presented in this work (see the Experimental Section).

Figure 2 shows the effect of the OEG grafting density on the growth regime (exponential, pseudo-linear, non-growing) of the (PLL-OEG/PSS) PEM films. The frequency shift $(\Delta f)$ of the 5 th overtone for the adsorbing layers was recorded by QCM-D and plotted against the number of deposited layers in
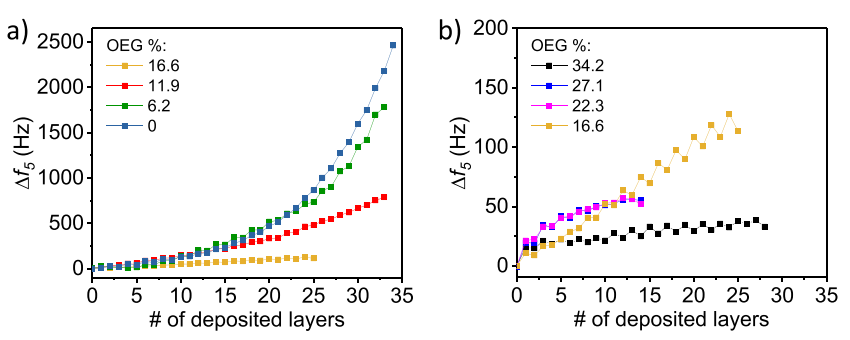

Figure 2. QCM-D frequency shifts (5th overtone) of the alternate deposition of PSS and modified PLL with (a) 0\% (cobalt blue), 6.2\% (green), 11.9\% (red), and 16.6\% (yellow) and (b) 16.6\% (yellow), $22.3 \%$ (purple), $27.1 \%$ (blue), and $34.2 \%$ (black) of grafted OEG. All adsorption steps were performed using $0.5 \mathrm{mg} / \mathrm{mL}$ of both polyelectrolyte solutions (PBS, $\mathrm{pH}$ 6.4). A step without flow and a washing one with PBS buffer were placed between each deposited layer (Figure S3). 
the PEM (the process of growth monitoring is shown in Figure S3; the full time traces are reported in Figure S4). For clarity, Figure $2 \mathrm{a}$ and $\mathrm{b}$ reports OEG grafting densities ranging from 0 to $16 \%$ and from 16 to $34 \%$, respectively (the data for $16.6 \%$ is present in both graphs for comparison). The layer formation was monitored up to 33 bilayers unless the growth was evidently linear or saturated before that.

It is known from literature that the density of ionized groups along the polymer chain and the charge mismatch can shift the type of growth between linear and exponential. ${ }^{33,60}$ Exponential growth is generally attributed to chain diffusion into the film material accompanying an adsorption step. ${ }^{54}$ Figure $2 \mathrm{a}$ shows the gradual transition between the growth regimes upon increasing the content of the grafted OEG. The increase in OEG groups grafted to the PLL reduces the amount of ionizable amino groups, which are largely positively charged at $\mathrm{pH} 6.4$ used in our experiments. We suppose that the insertion of the oligoethers as side chains blocks the interdiffusion, which is required for exponential growth, and thus causes pseudo-linear growth with increasing OEG density. Strongly exponential growth was recorded for the native PLL/PSS multilayer (blue line in Figure 2a), which is comparable to results reported earlier. ${ }^{35}$ By increasing the OEG grafting density of the PLL, their steric demand restricts the diffusion of adsorbed chains into the layer. As a consequence, the extent of exponential growth was progressively reduced from exponential (green and red lines, 6.2 and 11.9\% OEG) to linear (yellow line, $16.6 \% \mathrm{OEG}$ ). The sterically repulsive effect of OEG became evident at higher fractions of OEG-functionalized PLL (Figure 2b), where the linear regime for $16.6 \%$ PLL-OEG (yellow line) shifted to an only marginally growing regime for OEG mol fractions above 20\% (purple, blue, and black lines). At high contents of OEG, the steric repulsion caused by the densely ethylene glycol-functionalized layer was predominant over the charge overcompensation effect, which is essential for the $\mathrm{LbL}$ formation, ${ }^{34}$ resulting in little or almost zero polyelectrolyte adsorption.

In addition, structural information about the viscoelasticity of the growing PEM can be qualitatively assessed from the dissipation signal recorded by QCM-D. It has been reported that PLL/PSS films are stiffer than others such as PLL/HA ${ }^{35,53}$ or PLL/alginate. ${ }^{64}$ However, the introduction of the OEG moieties affected the viscoelasticity. Upon adsorption of PSS and PLL, asymmetric swelling steps could be identified from the dissipation changes $(\Delta D)$ (Figure $S 4 a-d$ ), while the PEMs quickly deflated when flowing PBS (without PE). In the case of high contents of OEG (Figure $S 4 \mathrm{e}-\mathrm{g}$ ), this $\Delta D$ asymmetry reduced between PLL-OEG and PSS layer depositions after the 2 nd bilayer. This is attributed to steric hindrance of the OEG units, which resulted in almost non-growing films. Overall, the $\Delta D$ in each measurement with OEG grafting densities above $10 \%$ was relatively small $\left(\sim 10^{-6}\right)$, confirming the increased rigidity and compactness of the layered structure. However, multilayers formed with native PLL showed differences between the $\Delta D$ overtones in the exponential regime (Figure S4a) in concert with high changes in $\Delta D$, which indicate a soft and swollen PEM. A similar effect was visible for low-grafted modified PLL (Figure S4b). Tuning the growth process is of utmost importance to express specific mechanical and structural properties of the PEM, which consequently may affect the probe density in DNA sensing. In that light, finding the proper combination of grafted OEG and $\mathrm{DBCO}$ is essential to simultaneously provide antifouling behavior and maximize the density of reactive groups for efficiently anchoring azido-DNA probes.

In order to study the effect of DBCO in the PEM formation, four types of PLL-OEG-DBCO polymers were tested to specifically target exponentially and linearly grown films while retaining a minimum amount of OEG. Figure 3 shows the $\Delta f$ (5th overtone) for each measurement plotted against the total number of layers (full time traces in Figures S5 and S6a). Little effects seemed to occur in the formation of viscoelastic PEMs, similar to that of native PLL (Figure S4a), when using modified PLL bearing 1.8 or $2.3 \%$ DBCO with a low OEG content (red and blue lines in Figure 3). The two other grafted densities gave the (pseudo-)linear and almost non-growing trends for PLL-OEG(8.5)-DBCO(1.8) and PLL-OEG(11.8)$\mathrm{DBCO}(1.8)$, respectively (yellow and green lines in Figure 3), as confirmed by the $\Delta D$ trends (Figure $S 5 b, c$ ) similar to the corresponding PLL-OEG (Figure S4c,d) with approximately the same grafting densities. Overall, these results indicate the possibility of targeting specific PEM growth regimes, showing the control of both physical (softness and hydrophilicity) and chemical (antifouling and types/amounts of reactive groups) properties of the PEM film by the proper design of the PLL component. For use in the probe binding systems, the PLLOEG(4.9)-DBCO(1.8) was found to be a good choice for the PEM formation, simultaneously having a significant DBCO content and showing exponential growth, while the PLLOEG(8.5)-DBCO(1.8), exhibiting linear growth, was used as a reference.

Enhanced Probe Binding and DNA Detection by Exponentially Growing PEMs. The main aim of this study is to investigate whether the type of growth regime can determine the effective probe density within the multilayer and thus affect the sensitivity in DNA detection. In the case of exponentially grown films, there are (at least) two ways by which the probe density can be improved over linearly grown films with the same number of layers. First, the mass of PE adsorbed in each step may increase, thus leading to enhanced loading of coupling groups (DBCO) when using functionalized PLL. Second, the accessibility toward DBCO, especially to those groups buried inside the PEM film, may be affected by the "openness" of the architecture. A compact, linearly grown layer will present a lower permeability for (bio)molecules, but the more hydrated and less dense nature of an exponentially grown PEM $^{35}$ may provide a better accessibility of the reactive groups. In addition, the retention effect of the analyte can be enhanced due to the "dense hydrogel" nature of the PEM. ${ }^{65}$ All in all, the combination of exponential growth and a high number of layers is expected to yield a higher loading of azidoDNA probes and concomitantly an improved sensitivity.

In order to investigate these effects, PLL-OEG(8.5)$\operatorname{DBCO}(1.8)$ and PLL-OEG(4.9)-DBCO(1.8) were exploited in PEM film formation with PSS to form linearly and exponentially grown multilayers, respectively (see Figure 3 above). Thereafter, DNA-azide probes were reacted to the DBCO groups of the PLL followed by the hybridization with cDNA. Figure $4 \mathrm{a}$ and $\mathrm{b}$ reports the $\Delta f$ (5th overtone) values respectively for the DNA probe immobilization and cDNA detection step (both $0.5 \mu \mathrm{M}$, PBS 6.4) as a function of the number of PLL-OEG-DBCO layers within the PEM. Differences are evident between the exponential (orange) and linear (green) regime (full measurements are reported in Figures S6 and S7). With the increasing number of layers, the probe incorporation and the consecutive DNA hybridization 


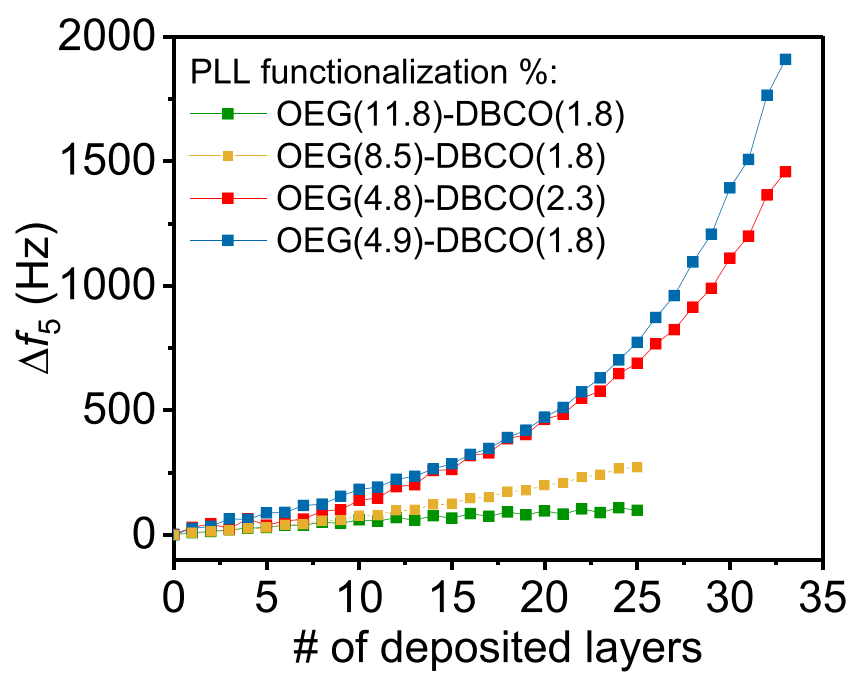

Figure 3. QCM-D frequency shifts (5th overtone) of the alternating deposition of PSS and PLL-OEG(4.9)-DBCO(1.8) (blue), PLLOEG(4.9)-DBCO(2.3) (red), PLL-OEG(8.5)-DBCO(1.8) (yellow), and PLL-OEG(11.8)-DBCO(1.8) (green). The adsorption steps were performed using $0.5 \mathrm{mg} / \mathrm{mL}$ of both modified PLL and PSS solutions (PBS, pH 6.4).
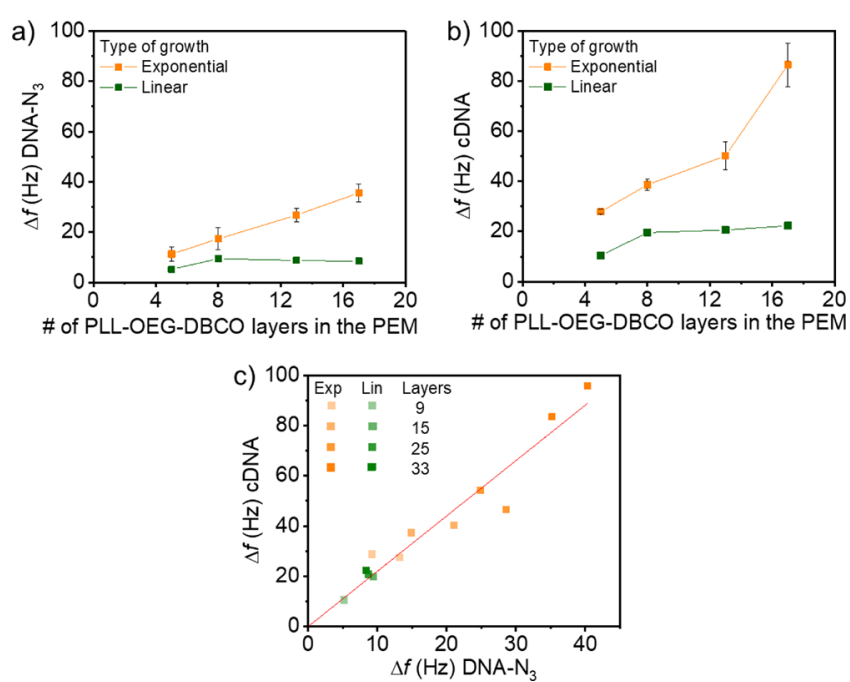

Figure 4. Dependence of $\Delta f$ (5th overtone) recorded by QCM for (a) DNA probe (23 nt) anchoring and (b) cDNA (43 nt) detection versus the number of PLL-OEG-DBCO in the PEM with exponential (orange) or linear (green) growth. (c) Frequency shifts (5th overtone) for DNA probe anchoring ( $x$-axis) and cDNA selective recognition ( $y$-axis) after the formation of PEMs. The color intensity scales with the number of layers in the PEM. The LbL process was performed using PSS and PLL-OEG(4.9)-DBCO(1.8) (orange points) or PLL-OEG(8.5)-DBCO(1.8) (green points). Raw data are reported in Figures S6 and S7. Data points for exponentially grown multilayer films represent two independent measurement series, while one series is shown for the linear PEM films.

increased linearly for the former (orange lines), while saturation was reached at the 8th bilayer (Figure 4a,b) for the latter (green lines). The $\Delta D$ changes in Figure $S 7$ indicate that this effect is probably due to the more compact film in the case of the linearly grown films, which blocks the penetration of incoming DNA, clearly indicating the different DNA sensitivity between the two types of architectures.
The type of architecture influenced both the DNA anchoring and detection, even though the lengths of the probe and target DNA were different. Figure $4 \mathrm{c}$ reports the $\Delta f$ values for the cDNA detection step as a function of the ones of DNA probe immobilization for exponentially (orange squares) or linearly (green squares) grown PEMs. The linear fit in Figure $4 \mathrm{c}$ shows the linear response effect of binding $\mathrm{cDNA}$ as a function of the probe density. The value of the slope of the fit in Figure $4 c$, normalized for the nucleotide number of the probe and cDNA, gave a hybridization efficiency of $125 \%$ for both growth regimes. This apparent overestimation of the hybridization efficiency has already been reported in literature, and it is associated with differences in DNA length and the formation of double-stranded DNA species, which cause a change in the degree of hydration. ${ }^{66,67}$ Overall, we can conclude that all available DNA probes in the PEM films can be hybridized by cDNA.

The amplification effect of an exponentially grown multilayer is clearest in the comparison with a monolayer of functionalized PLL (Figure S8). The $\Delta f$ values for both the probe and cDNA steps of the 33-layer exponentially grown PEM were approx. 25 times higher than those of a monolayer of PLLOEG(29.7)-DBCO(1.6) (a larger amount of OEG was required to avoid non-specific interaction of DNA to the PLL backbone in this case, ${ }^{68}$ as shown in the left part of Figure S8). In the case of a linearly grown PEM, the frequency changes recorded at the QCM for the same DNA probe and cDNA incorporation were only a factor of 5.5 higher. This data illustrates the sensitivity enhancement of the multilayer approach and especially of PEMs that are exponentially grown, confirming that the probe density can be tuned by exploiting exponentially grown films, while a saturation of the probe density occurs for linearly grown films.

The selectivity of the DNA biorecognition process was tested for all the exponentially grown multilayers by flushing a solution of non-complementary DNA (ncDNA) $(0.5 \mu \mathrm{M}, \mathrm{PBS}$ 6.4) before the complementary one (Figure $\mathrm{S} 6 \mathrm{a}-\mathrm{h}$ ). Adsorption of ncDNA was absent in all systems (no $\Delta f$ shifts visible upon injection), regardless of the total number of layers. Additionally, other tests were performed to investigate the influence of DBCO on the DNA selectivity, using two PEMs of the exponentially and linearly grown architectures consisting of 9 layers of PLL-OEG(6.2) or PLL-OEG(11.9) (thus, in the absence of DBCO groups, Figure S9a,b), respectively. The lack of non-specific adsorption for the consecutive flows of DNAazide, ncDNA, and target cDNA (Figure S9b,d) further confirmed the overall antifouling behavior of the PEM films given by the combination of OEG and zwitterionic-like motifs.

A key point for the amplified DNA detection is to what extent the DBCO groups present in the PEM films react with the probe DNA. Not all functional groups within the PEM structure may participate in the binding and recognition process. In order to study this effect, two PEM structures with PLL-OEG but just one PLL-OEG(4.9)-DBCO(1.8) layer either as the last or first deposited layer were exploited (Figure S10). When the PLL with appended DBCO was used on top of the PEM, $\Delta f$ values of roughly 5.5 and $12.5 \mathrm{~Hz}$ were observed for $\mathrm{DNA}_{3} \mathrm{~N}_{3}$ and $\mathrm{cDNA}$, respectively, while no significant frequency changes were found for PLL-OEG(4.9)$\operatorname{DBCO}(1.8)$ as the first layer, suggesting that more deeply buried layers are less accessible for the probe binding and DNA recognition process. 

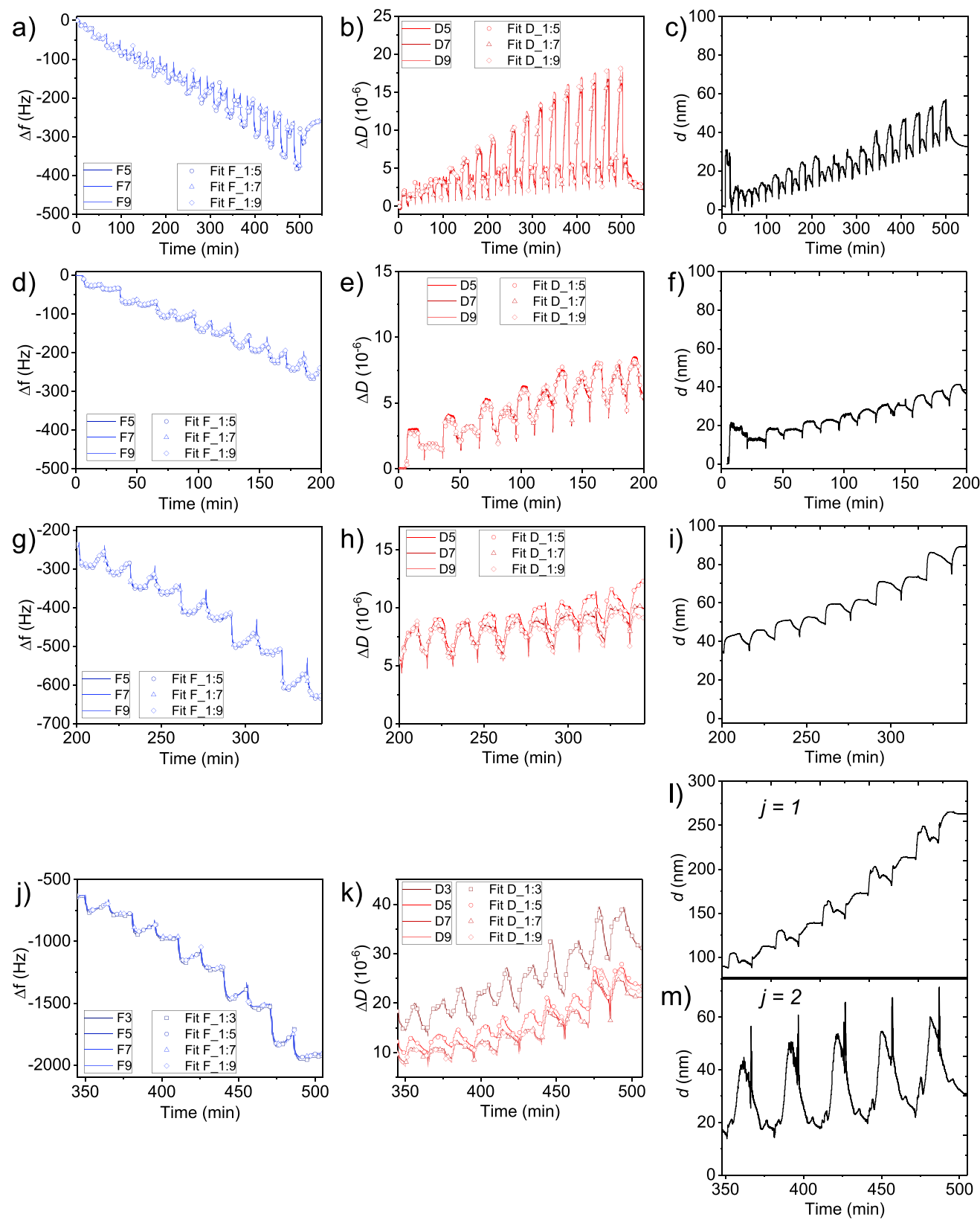

Figure 5. Fitting and modeled thicknesses of the LbL parts of the 33-layer PEMs for the $(\mathrm{a}-\mathrm{c})$ linear and $(\mathrm{d}-\mathrm{m})$ exponential regimes, using the PLL-OEG(8.5)-DBCO(1.8) and PLL-OEG(4.9)-DBCO(1.8) polymers, respectively. $(\mathrm{a} / \mathrm{d} / \mathrm{g} / \mathrm{j}) \Delta f$ and $(\mathrm{b} / \mathrm{e} / \mathrm{h} / \mathrm{k}) \Delta D$ time traces and best fits (symbols and lines, respectively) for the 5 th-9th overtones (the 3rd one is included $(\mathrm{j}, \mathrm{k})$ in the case of a two-overlayer fit $(j=1)$ with $(\mathrm{c} / \mathrm{f} / \mathrm{i} / \mathrm{l} / \mathrm{m}$ ) the modeled thicknesses). The best fits were obtained with $(a-i)$ one $(j=1)$ or $(j-m)$ two $(j=1,2)$ overlayers. The Voigt-based model was used with fixed densities of $(\mathrm{a}-\mathrm{c}) 1.20 \mathrm{~g} / \mathrm{cm}^{3},(\mathrm{~d}-\mathrm{f}) 1.12 \mathrm{~g} / \mathrm{cm}^{3},(\mathrm{~g}-\mathrm{i}) 1.20 \mathrm{~g} / \mathrm{cm}^{3}$, and $(\mathrm{j}-\mathrm{m}) 1.20 \mathrm{~g} / \mathrm{cm}^{3}(j=1)$ and $1.05 \mathrm{~g} / \mathrm{cm}^{3}(j=2)$. The fit results for the shear modulus and viscosity are shown in Figure S11.

Evaluation of the Viscoelasticity of the PEMs and Thickness Determination. Important questions regarding the interpretation of the QCM data involve quantitative changes of physicochemical parameters of the PEMs as a function of the layer number, film thickness, and growth regime. In order to evaluate the thickness and the mass increment of both the LbL formation and DNA anchoring/ hybridization steps, a Voigt model based on the general case of two individual regions of the film with different viscoelastic properties (overlayers) was applied. ${ }^{69}$ The PEM formation and DNA insertion processes of four PEM architectures, specifically those with 9 and 33 layers, each in the linear and exponential growth regimes, were treated separately as one $(j=$
1) or two $(j=1,2)$ overlayers (where $j$ expresses the overlayer number in the model, starting from the substrate). A more detailed description of the Voigt-based modeling analysis is given in the Supporting Information.

Figure 5 shows the fitting (open symbols) of the $\Delta f$ and $\Delta D$ data (blue and red lines) for the time span of LbL formation for the 33-layer PEMs with high OEG density (linear growth, Figure $5 a-c$ ) and low OEG density (exponential growth, Figure $5 \mathrm{~d}-\mathrm{m}$ ), according to eqs $\mathrm{S} 7$ and S8. The linearly grown multilayer time span (Figure $5 a-c$ ) was properly fitted using a single overlayer on top of the piezoelectric chip $(j=1)$, with a density of $1.2 \mathrm{~g} / \mathrm{cm}^{3}$. Table S3 lists all densities that have been used as fixed input parameters to fit each time span of the 

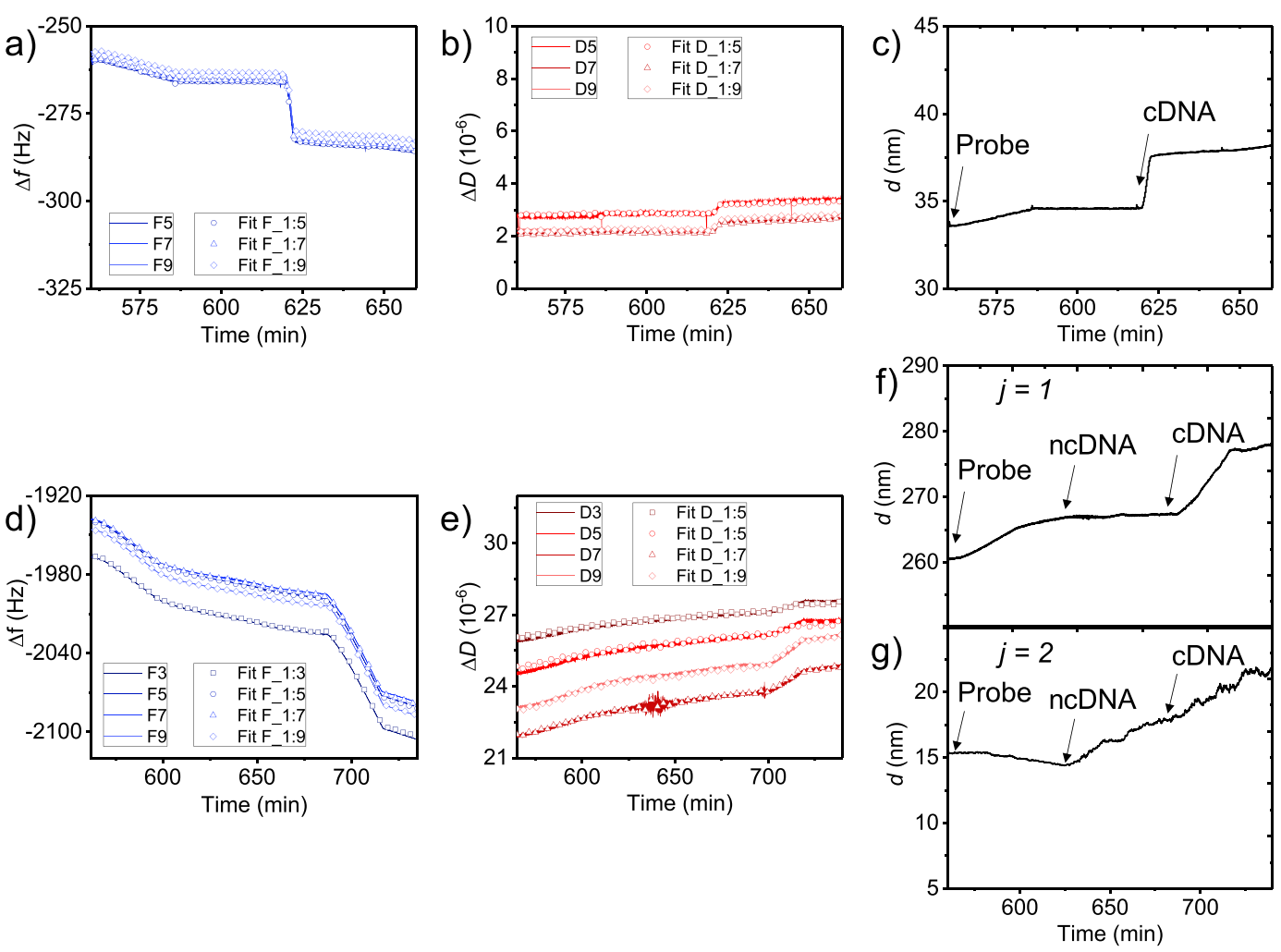

Figure 6. Fitting and modeled thicknesses of the DNA part of the 33-layer PEMs made in $(a-c)$ linear and $(d-g)$ exponential regimes, using the PLL-OEG(8.5)-DBCO(1.8) and PLL-OEG(4.9)-DBCO(1.8) polymers, respectively. (a/d) $\Delta f$ and (b/e) $\Delta D$ time traces and best fits (symbols and lines, respectively) for the 5th-9th overtones (the 3rd one is included (d,e) in the case of a two-overlayer fit $(j=1,2)$ with $(\mathrm{c} / \mathrm{f} / \mathrm{g})$ the modeled thicknesses). The best fits were obtained with $(\mathrm{a}-\mathrm{c})$ one $(j=1)$ or $(\mathrm{d}-\mathrm{g})$ two $(j=1,2)$ overlayers. The Voigt-based model, checked for robustness, was used with fixed densities of $(\mathrm{a}-\mathrm{c}) 1.07 \mathrm{~g} / \mathrm{cm}^{3}$ and $(\mathrm{d}-\mathrm{g}) 1.12 \mathrm{~g} / \mathrm{cm}^{3}(j=1)$ and $1.07 \mathrm{~g} / \mathrm{cm}^{3}(j=2)$. The results for the shear modulus and viscosity are shown in Figure S14.

different PEM architectures with either the one- or twooverlayer model $(j=1$ or $j=1,2)$. All fitted parameter values reported were checked for robustness (see the Supporting Information). As a result, all fitted values of the layer thickness $(d)$, shear modulus $(\mu)$, and viscosity $(\eta)$ increased linearly with the number of layers (Figure $5 \mathrm{a}-\mathrm{c}$ and Figure $\mathrm{S11} \mathrm{a}, \mathrm{b}$ ). The relatively low increment per deposited layer (compared to the exponential PEM) is a sign of a rigid multilayer structure, as further confirmed by the absence of splitting between the overtones for both $\Delta f$ and $\Delta D$ and the small changes in the latter upon adsorption of modified PLL (Figure 5a,b). The same density was found to be optimal for the one-overlayer $(j=1)$ model of the 9-layer PEM in the linear regime (Figure S12), with the resulting values for the shear modulus, thickness, and viscosity similar to the corresponding values obtained from the first 9-layer section of the 33-layer linear PEM (Figure 5c and Figure S11a,b versus Figure S12c-e). This confirms the interpretation of a rigid, thin multilayer architecture.

The LbL formation time span in the exponential PEM with 33 layers had a more complex evolution, causing the inability to find a proper fit using the one- or two-overlayer model. This effect is ascribed to the occurrence of either more than two overlayers, and densities, or a gradient of densities and/or viscoelastic parameters along the surface normal of the PEM. Looking at the full time trace in Figure S6, three time frames could be defined by the dissipation signal, as indicated by the separate representation in Figure 5d,e (0-200 $\mathrm{min})$, Figure $5 \mathrm{~g}, \mathrm{~h}(200-345 \mathrm{~min})$, and Figure 5j,k (345-510 min). The first time frame ( 11 layers, Figure $5 \mathrm{~d}, \mathrm{e}$ ), with growing $\Delta D$, was fitted with a lower density $\left(\rho=1.12 \mathrm{~g} / \mathrm{cm}^{3}\right)$ than the linear 33layer $\operatorname{PEM}\left(\rho=1.20 \mathrm{~g} / \mathrm{cm}^{3}\right)$, suggesting a softer, more hydrated PEM. Similar values for the fit parameters $(d, \mu$, and $\eta$ ) were obtained from the fitting of the LbL time span of the 9-layer PEM in the exponential regime (Figure S13) using the same density of $1.12 \mathrm{~g} / \mathrm{cm}^{3}$ (one-overlayer model, $j=1$ ), confirming the pronounced viscoelastic nature of the exponentially grown architecture. In the second time frame (up to the 23rd layer, Figure 5g,h), an average constant dissipation, typical of PEMs exhibiting linear growth, indicated a "compression" of the exponentially growing PEM. Thus, this second time frame was modeled using just one overlayer $(j=$ 1) with a higher input density, equal to the linearly growing PEMs $\left(\rho=1.20 \mathrm{~g} / \mathrm{cm}^{3}\right)$. Finally, the third time frame (Figure $5 \mathrm{j}, \mathrm{k})$, in which the exponential effect was most evident, could not be modeled by a single homogeneous overlayer $(j=1)$, most likely due to a change of density along the PEM. Here, the two-overlayer $(j=1,2)$ model was exploited with densities of $1.20 \mathrm{~g} / \mathrm{cm}^{3}(j=1$, equal to the density of the 2 nd time frame) and $1.05 \mathrm{~g} / \mathrm{cm}^{3}(j=2$, in between the density of the 1 st time frame and the effective density of a PLL-PEG monolayer reported in literature, $\left.\rho=1.027 \mathrm{~g} / \mathrm{cm}^{3}\right){ }^{70}$ The presence of a second "adsorbing" layer, namely, the overlayer $j=2$, was evident from the results of the fitting from the third time frame (Figure 51,m and Figure $\mathrm{S} 11 \mathrm{~g}-\mathrm{j}$ ). The shear modulus and viscosity of the $j=1$ overlayer increased approx. by a factor of 4 during the depositions, while their values were about 20 times larger than those reported for a $(\mathrm{PLL} / \mathrm{HA})_{19}$ multilayer 
Table 1. Calculated Thicknesses $(d)$ and Estimated Surface Densities for Modified PLL and PEM Architectures, DBCO within the Whole PEM and from the Last Layer, and DNA Probe/Target cDNA Using the Densities of the Voigt-Based Model for the 9- and 33-Layer PEM Architectures, Each with Linear and Exponential Growth ${ }^{b}$

\begin{tabular}{|c|c|c|c|c|}
\hline & \multicolumn{4}{|c|}{ number of layers } \\
\hline & \multicolumn{2}{|c|}{9} & \multicolumn{2}{|c|}{33} \\
\hline & linear & exponential & linear & exponential \\
\hline$d_{\mathrm{PLL}}\left[\right.$ total $\left.d_{\mathrm{PEM}}\right](\mathrm{nm})-d_{\mathrm{PLL}} / d_{\mathrm{PEM}}(\%)^{a}$ & $11.7[16]-73 \%$ & $18.3[24]-76 \%$ & $25.5[36]-71 \%$ & $167.3[290]-57 \%$ \\
\hline $\mathrm{DBCO}$ in all PEM (molecules $/ \mathrm{cm}^{2}$ ) & $1.7 \times 10^{13}$ & $2.5 \times 10^{13}$ & $3.6 \times 10^{13}$ & $2.3 \times 10^{14}$ \\
\hline $\mathrm{DBCO}$ from last layer (molecules $/ \mathrm{cm}^{2}$ ) & $7.1 \times 10^{11}$ & $4.0 \times 10^{11}$ & $3.6 \times 10^{12}$ & $4.0 \times 10^{13}$ \\
\hline DNA probe (molecules $/ \mathrm{cm}^{2}$ ) & $1.4 \times 10^{12}$ & $2.0 \times 10^{12}$ & $1.7 \times 10^{12}$ & $1.1 \times 10^{13}$ \\
\hline target cDNA (molecules $/ \mathrm{cm}^{2}$ ) & $2.4 \times 10^{12}$ & $4.3 \times 10^{12}$ & $2.8 \times 10^{12}$ & $1.9 \times 10^{13}$ \\
\hline
\end{tabular}

${ }^{a_{T}}$ The values in squared brackets refer to the total thickness in nm of the whole PEM structure (modified PLL/PSS) followed by the estimated percentage (\%) of thickness attributed only to the modified PLL polymer in the same PEM architecture. ${ }^{b}$ See the Experimental Section. Water (80\%) was assumed to be present for the calculated masses (modified PLL and DNA species).

film with non-functionalized PLL, ${ }^{62}$ suggesting that the OEG content strongly affected the internal properties of the PEM. Interestingly, the second overlayer $(j=2)$ of approx. $20 \mathrm{~nm}$ thickness acted like a "buffering" top region, where $d, \mu$, and $\eta$ did not strongly depend on the number of deposited layers. Therefore, this top layer can be seen as a diffusion zone with constant size that promotes the exponential growth, as explained by Porcel and coworkers. ${ }^{61}$

To investigate the amplification effect of exponential PEMs on the DNA probe (23 nt) immobilization and the following cDNA (43 nt) recognition, the DNA anchoring/detection steps on 33-layer PEMs, either in the linear or exponential growth regime, were modeled using the one- (linear) or two(exponential) overlayer model. Figure 6 shows the fitted time traces of the frequency and dissipation signals for the linear (Figure 6a,b) and exponential (Figure 6d,e) 33-layer structures, together with the respective modeled thicknesses (Figure $6 \mathrm{c}, \mathrm{f}$, and $\mathrm{g}$ ). The best fit for the DNA part in the linearly grown PEM was achieved with one overlayer $(j=1)$ with a density of $1.07 \mathrm{~g} / \mathrm{cm}^{3}$. This value was fixed due to the optimized density of the LbL formation for the same PEM $\left(\rho=1.20 \mathrm{~g} / \mathrm{cm}^{3}\right)$, the one of pure water $\left(1.00 \mathrm{~g} / \mathrm{cm}^{3}\right)$, and the one of DNA $\left(1.06 \mathrm{~g} / \mathrm{cm}^{3}\right)$ detected by QCM on a supporting lipid bilayer. ${ }^{66,71}$ It is interesting to note that while the addition of the DNA probe affected the frequency (Figure 5a, $560 \mathrm{~min}$ ), no significant changes were recorded for the dissipation (Figure 5b). Moreover, the corresponding viscoelastic properties were almost not altered by any of the DNA molecules (Figure S14), further confirming the stiff nature of the PEM film. The same behavior was found for the DNA anchoring/ detection in the case of the 9-layer PEM with linear growth (Figure S15), suggesting that the DNA probe was immobilized onto the last layer(s), without diffusion into the PEM.

On the other hand, the fitting of the DNA steps in the exponentially grown 33-layer PEM provided a different situation (Figure $6 \mathrm{~d}-\mathrm{g}$ and Figure $\mathrm{S} 14 \mathrm{c}-\mathrm{f}$ ). The fitting was only possible using a two-overlayer model $(j=1,2)$ with different densities of $1.12 \mathrm{~g} / \mathrm{cm}^{3}$ (in between the densities found for the LbL part in the exponential 33-layer PEM) and $1.07 \mathrm{~g} / \mathrm{cm}^{3}$. This approach was chosen because similarly for the 9-layer PEM with exponential growth, the DNA insertion step could only be fitted with the two-overlayer model, exploiting the same densities (Figure S16). The behavior of DNA probe anchoring and the cDNA detection was significantly different between the fitted overlayers $(j=1$ and $j=2$ ) for the 33-layer architecture. Upon immobilization of the DNA probe to the DBCO moieties, the thickness and viscosity of the $j=1$ overlayer were more affected, in comparison with the overlayer in contact with the bulk liquid $(j=2)$ (Figure $6 \mathrm{f}, \mathrm{g}$ and Figure S14c-f). The shear modulus did not change substantially, suggesting that the DNA-azide binding had little effect on both overlayers. However, the hybridization with cDNA to form double-stranded DNA had a major impact on the first overlayer. The higher frequency change (Figure 6d), and indeed the thickness (Figure 6f), of cDNA is probably related to a more open structure and a deeper penetration of cDNA compared to every other PEM architecture analyzed, resulting in more uptake of water. To support this concept, it has to be noted that for the linearly grown architectures, a saturation of cDNA within the PEM occurred right after the injection of the solution (around $620 \mathrm{~min}$, Figure 6a), increasing the thickness by just $3 \mathrm{~nm}$ (Figure 6c), compared to the $13 \mathrm{~nm}$ of the 33-layer exponential PEM. At the same time, the viscosity was reduced, while the shear modulus increased, defining a fluid-like but more rigid PEM (Figure S14c,d). These effects could be ascribed to the DNA hybridization, making the structure stiffer, while the hydration shell of double-stranded DNA made it more gel-like. ${ }^{36,38}$

The fitted overlayer $j=2$ of exponentially grown PEMs showed a remarkable difference in thickness, being 5 and 20 $\mathrm{nm}$ for 9 and 33 layers, respectively. A peculiar effect was found for the 9-layer exponential architecture upon hybridizing cDNA and washing with PBS, in which all fitted parameters $(d$, $\mu$, and $\eta$ ) increased (Figure S16f-h). The 33-layer multilayer film reported a similar but gradual increment when ncDNA (42 nt) was injected (Figure $6 \mathrm{~g}$ and Figure S14e,f), suggesting diffusion of the DNA solely inside the "buffering" zone (overlayer $j=2$ ), affecting its structure. Notably, the ncDNA appeared to not hybridize to the DNA probe or penetrate further, as shown from the absence of changes in the viscoelastic parameters of the first fitted overlayer $j=1$ and all overtones (Figure $6 \mathrm{~d}-\mathrm{f}$ and Figure $\mathrm{S} 14 \mathrm{c}, \mathrm{d}$ ). This behavior also confirmed the soft gel state in particular of the exponential PEM, having an enhanced permeability and retention effect, demonstrating the capability of controlling the viscoelastic properties and the openness of multilayer structures, resulting in amplified anchoring and detection of DNA molecules.

Quantification of Coupling and Hybridization Efficiencies. In order to assess the amplification sensitivity due to the effect of exponential growth, the densities used to model the four PEM structures (Table S3) were exploited to estimate the amounts of modified PLL adsorbed, consequently, the corresponding DBCO surface densities, and the surface densities of the DNA probe and hybridized cDNA molecules. 
In particular, the densities in Table S3 were used to derive the Voigt mass, defined as the coupled surface mass coverage from the model $(m=\rho \times d)$, of each time span (LbL formation and DNA insertion). However, the Voigt mass does not distinguish between the dry mass of the molecules and the associated water. $^{72,73}$ Consequently, two approaches were used for estimating the coverage of adsorbed molecules from the thickness data. Davila et al. quantified the amount of hydration water for ethylene glycol-functionalized poly(acrylic acid) (PAA) adsorbing on PEMs using QCM and SPR. ${ }^{47}$ For PAAs with 5 and $10 \%$ of the monomers grafted with a linker having three ethylene glycol units end-functionalized with biotin moieties, the degrees of hydration were approx. 83 and $80 \%$, respectively. Alternatively, we used ellipsometry to obtain the dry mass of the PEM films (Table S4). While the data show a good qualitative comparison across the four samples with the mass coverages obtained by QCM, directly comparing the mass densities to obtain hydration water fractions led to degrees of hydration ranging from 23 to 63\%, with both exponential layers having values around $60 \%$ (Table S5).

When assuming that $80 \%$ of the surface mass coverage from the PLL-OEG-DBCO, as well as from single and doublestranded DNA species, corresponds to hydration water, ${ }^{50,66,73}$ the surface coverages of the respective species were calculated (Table 1). Discrepancies between the values for probe DNA and cDNA molecules could be ascribed to the different hydration shells when referring to single- or double-stranded DNA molecules. ${ }^{72,73}$

Overall, the data shown in Table 1 indicates that the difference between linearly and exponentially grown films is not yet significant in the case of 9 bilayers (all DBCO and DNA densities approx. equal), but it becomes much enhanced at 33 bilayers (data for the exponential film an order of magnitude larger). The total projected density of DBCO within the whole PEM was higher when increasing the number of layers and when having exponential growth. However, the exponentially grown structure with 33 layers has a major contribution to the projected DNA density from the last layer, which is two orders of magnitude higher than the corresponding last layer in the exponential 9-layer PEM. As shown in Figure 4, the amounts of probe DNA and cDNA detected for the linearly grown PEM structures were similar regardless of the number of layers. On the other hand, the 9layer exponential PEM had a higher amount for both DNA species compared to the PEM architectures with linear growth, validating our assumption that exponentially grown PEMs display a more open structure. The exponentially grown PEM with 33 layers has a reduced contribution of PLL to the total thickness (only 57\% from PLL, Table 1), suggesting more adsorption from PSS to enhance the exponential growth mode. Overall, the efficiency of probe binding appeared to be similar for all the PEMs analyzed. A 7-times increment was found for the 33-layer exponentially grown PEM compared to the linear one with the same number of layers, indicating that the mass adsorbed in the last layer has a stronger effect on the probe binding efficiency. These data also shows the increased amount of detected cDNA, confirming the capability of enhancing the sensitivity of DNA detection by exploiting purposefully engineered open structures.

\section{CONCLUSIONS}

In summary, we have presented an amplification strategy for DNA recognition exploiting exponentially grown PEM architectures with appended antifouling and clickable groups at the interface. The hybridization efficiency was retained for all the investigated systems, confirming that the increased loading and selective detection of DNA in the exponentially grown PEM films were related to the more open architecture.

By making use of a Voigt-based model, the viscoelastic properties of four model multilayer structures were assessed, further indicating the more elastic, gel-like characteristics of the exponentially grown PEMs. The modeled thicknesses were exploited to evaluate the effectiveness of the probe anchoring and consecutive DNA hybridization, indicating an increase in DNA probes immobilized inside the multilayer structure for the 33-layer exponentially grown PEM, which resulted in a 25fold amplification of the detected signal compared to a PLL monolayer with the same grafting density of DBCO groups.

The amplification strategy by the structural design of the biosensing interface has shown to improve the detection of a model DNA target by extending the detectability of the binding events into the third dimension. Such a bio-orthogonal approach is not bound to gravimetric analysis and can be exploited for several other surface-based detection systems, for example, surface plasmon resonance (SPR) or enzyme-linked immunosorbent assay (ELISA). Because of the LbL modification strategy and the customizable PLL, the physical and chemical characteristics of the biorecognition interface can be tailored, indicating the potential universality of such a surface-engineering approach with intrinsic amplification properties.

All in all, gravimetric, optical, and electrochemical-based biosensors can ultimately benefit from this strategy due to the physical extension of the transducing interface. Such 3D, biocompatible gel-like structures may enhance the detection of multiple, charged, and biologically relevant molecules, such as nucleotides, proteins, and drugs, further improving the production of personalized diagnostic devices and point-ofcare recognition systems.

\section{EXPERIMENTAL SECTION}

Materials. Poly-L-lysine hydrobromide ( $M W=15-30 \mathrm{kDa}$ by viscosity), poly(sodium 4-styrenesulfonate) (average $\mathrm{MW}=70 \mathrm{kDa}$ ), $\mathrm{D}_{2} \mathrm{O}$, and tablets for $10 \mathrm{mM}$ PBS solution ( $\mathrm{pH}$ 7.4, ionic strength of $150 \mathrm{mM}$ ) were obtained from Sigma-Aldrich. $\mathrm{HCl}$ solution was obtained from SelectiPur. Methyl-OEG ${ }_{4}-\mathrm{NHS}$ ester was purchased from ThermoFisher Scientific, while $\mathrm{DBCO}-\mathrm{OEG}_{4}-\mathrm{NHS}$ was obtained from Click Chemistry Tools. The membranes for dialysis (Spectra/Por, 6-8 kDa cutoff, diameter of $6.4 \mathrm{~mm}$ ) were purchased from Spectrum Labs, Greece. The azide-DNA (MW, $7421 \mathrm{~g} / \mathrm{mol} ; 5^{\prime}$ $\mathrm{N}_{3}$ - PEG $_{4}$-ACCACAAGTTTATATTCAGTCAT-3', $23 \mathrm{nt}$ ) was acquired from Biomers.net $\mathrm{GmbH}$ (chemical structure of the linker in Figure S1). cDNA (complementary to the KRAS sequence, 5'ATGACTGAATATAAACTTGTGGTAGTTGGAGCTGGTGGCGTAG-3', 43 nt) and ncDNA (5'-CTACGCCACCTCAACCTACGCCACCTCCACCTACGCCACCTC-3', $42 \mathrm{nt}$ ) were purchased from Eurofins Genomics and used as received.

Synthesis and Quantification of PLL with Appended Groups. The synthesis of PLL-OEG and PLL-OEG-DBCO polyelectrolytes and the quantification of OEG and DBCO mol fractions grafted to the PLL backbone were done by following previously reported procedures. ${ }^{25,50}$ Briefly, $10 \mathrm{mg} / \mathrm{mL}$ PLL HBr was dissolved in PBS 7.4, and stoichiometric amounts of Methyl-OEG $4^{-}$ NHS and DBCO-OEG ${ }_{4}$ NHS (both dissolved in DMSO at a concentration of $250 \mathrm{mM}$ ) were added simultaneously. After $4 \mathrm{~h}$ under stirring, the solution was dialyzed with a Spectra/Por dialysis membrane (molecular cutoff of 6 to $8 \mathrm{kDa}$ ) against decreasing concentrations of PBS buffer in Milli-Q water, including a full $24 \mathrm{~h}$ cycle in Milli-Q water. The final solution was freeze-dried overnight. 
The characterization of the final product was done by ${ }^{1} \mathrm{H}$ NMR (Figure S2), and it was stored at $-20{ }^{\circ} \mathrm{C}$ in Milli-Q water. Quantification of the grafted percentages has been extensively reported in the Supporting Information.

Quartz Crystal Microbalance Experiments. Silica-coated (50 nm, QSX303) QCM-D chips from LOT-Quantum were cleaned by rinsing them with Milli-Q water (Millipore) and EtOH. Thereafter, the chips were incubated for $1-2 \mathrm{~h}$ under stirring in aqueous solution of $2 \%$ SDS and then washed extensively with Milli-Q water and EtOH. After drying under nitrogen flow and oxidation with oxygen plasma (Plasma Prep II, SPI Supplies; 200-230 mTorr, $40 \mathrm{~mA}$ ) for $1.5 \mathrm{~min}$, the chips were mounted in the chambers and equilibrated with PBS ( $\mathrm{pH} 6.4)$. Solutions $(0.5 \mathrm{mg} / \mathrm{mL}$ ) of modified PLL and PSS in PBS ( $\mathrm{pH}$ 6.4) were alternatively injected at a flow rate of $120 \mu \mathrm{L} /$ $\mathrm{min}$ for $5 \mathrm{~min}$. The adsorption was continued for $5 \mathrm{~min}$ without flow followed by a $5 \mathrm{~min}$ rinsing with the same PBS buffer, again with a flow rate of $120 \mu \mathrm{L} / \mathrm{min}$. This procedure was used for all the $\mathrm{LbL}$ buildups, unless stated otherwise. After the LbL buildup, before the DNA insertion process, PBS buffer solution was flushed for approx. 1 $\mathrm{h}$ to equilibrate the PEM architecture. A second flow rate of $80 \mu \mathrm{L} /$ min was exploited during the DNA anchoring and hybridization steps. All the DNA species (probe, ncDNA, and cDNA) had a concentration of $0.5 \mu \mathrm{M}$ in PBS solution ( $\mathrm{pH}$ 6.4). The monolayer experiment was performed by adsorbing the functionalized PE PLLOEG(29.7)-DBCO(1.6) followed by the selective DNA anchoring and hybridization exploiting the same solution conditions and concentrations previously adapted in the PEM measurements (flow rate of $80 \mu \mathrm{L} / \mathrm{min}$ for each step). The QCM-D measurements were performed using a Q-Sense E4 4-channel quartz crystal microbalance with a peristaltic pump (Biolin Scientific), monitoring the fundamental overtone from 3rd to 9th. All experiments were performed in standard PBS buffer solution (10 $\mathrm{mM}$ phosphate, ionic strength of $150 \mathrm{mM}$, and $\mathrm{pH} 6.4$ ) at $21{ }^{\circ} \mathrm{C}$. The viscoelastic modeling is discussed in the Voigt-based analysis section in the Supporting Information.

Ellipsometry. Silicon substrates were activated in oxygen plasma for $1.5 \mathrm{~min}$, producing a $\mathrm{SiO}_{2}$ layer with $1.4 \mathrm{~nm}$ thickness, and functionalized by adapting the procedure of a QCM by alternatively dipping them in either PLL-OEG(4.8)-DBCO(1.8) or PLLOEG(8.5)-DBCO(1.8) solutions $(0.5 \mathrm{mg} / \mathrm{mL}$ in PBS 6.4) for 10 $\mathrm{min}$. Thereafter, the substrates were placed in Milli- $Q$ water for $5 \mathrm{~min}$ followed by dipping in PSS solution $(0.5 \mathrm{mg} / \mathrm{mL}$ in PBS 6.4) for another $10 \mathrm{~min}$. These steps were repeated until the desired number of layers was reached. The thickness of the multilayer architecture was measured by ellipsometry (Woollam M-2000UI) in the range of 245$1690 \mathrm{~nm}$, with spectral resolutions of $1.6 \mathrm{~nm}(245-100 \mathrm{~nm})$ and 3.2 $\mathrm{nm}(1000-1690 \mathrm{~nm})$ and a beam diameter of $300 \mu \mathrm{m}$. The ellipsometry data (values given are averages over 12 spots on the surface) were obtained at an incident angle of $75^{\circ}$ and fitted with a Cauchy layer with a refractive index of 1.46. A second set of measurements was performed after overnight drying at $60{ }^{\circ} \mathrm{C}$ in a controlled atmosphere setup. The values are reported in Table S4.

\section{ASSOCIATED CONTENT}

\section{SI Supporting Information}

The Supporting Information is available free of charge at https://pubs.acs.org/doi/10.1021/acs.chemmater.0c02454.

Chemical, synthesis and characterization of functionalized PLL polyelectrolytes, QCM and ellipsometry data acquisition, chemical structure of the azido-DNA linker, $1 \mathrm{H}$ NMR spectra of PLL-OEG-DBCO and PLL-OEG, visual explanation of LbL buildup at QCM, QCM data of the PEM formation and DNA insertion processes, DNA detection on monolayer of PLL-OEG-DBCO, selectivity and control experiments, fitting and modeled results of the PEM formation and DNA insertion processes, and Voigt-based modeling analysis (PDF)

\section{AUTHOR INFORMATION}

\section{Corresponding Authors}

Monika Schönhoff - Institute of Physical Chemistry, and Center for Soft Nanoscience, University of Münster, 48149 Münster, Germany; Email: schonhoff@uni-muenster.de

Jurriaan Huskens - Molecular NanoFabrication group, MESA+ Institute for Nanotechnology, Faculty of Science and Technology, University of Twente, 7500 AE Enschede, The Netherlands; ○ orcid.org/0000-0002-4596-9179; Email: j.huskens@ utwente.nl

\section{Authors}

Jacopo Movilli - Molecular NanoFabrication group, MESA+ Institute for Nanotechnology, Faculty of Science and Technology, University of Twente, 7500 AE Enschede, The Netherlands

Salmeen Shakil Choudhury - Molecular NanoFabrication group, MESA+ Institute for Nanotechnology, Faculty of Science and Technology, University of Twente, 7500 AE Enschede, The Netherlands

Complete contact information is available at:

https://pubs.acs.org/10.1021/acs.chemmater.0c02454

\section{Notes}

The authors declare no competing financial interest.

\section{ACKNOWLEDGMENTS}

The Horizon 2020 Health project "ULTRAPLACAD" (nr. 633937 ) is acknowledged for the financial support. Dr. Melissa J. Goodwin is acknowledged for her contribution in the ellipsometry experiments.

\section{REFERENCES}

(1) Jones, A. M.; Grossmann, G.; Danielson, J. Å.; Sosso, D.; Chen, L. Q.; Ho, C. H.; Frommer, W. B. In Vivo Biochemistry: Applications for Small Molecule Biosensors in Plant Biology. Curr. Opin. Plant Biol. 2013, 16, 389-395.

(2) Fang, Y. Label-Free Biosensors for Cell Biology. Int. J. Electrochem. 2011, 2011, 1-16.

(3) Kirsch, J.; Siltanen, C.; Zhou, Q.; Revzin, A.; Simonian, A. Biosensor Technology: Recent Advances in Threat Agent Detection and Medicine. Chem. Soc. Rev. 2013, 42, 8733-8768.

(4) Amine, A.; Mohammadi, H.; Bourais, I.; Palleschi, G. Enzyme Inhibition-Based Biosensors for Food Safety and Environmental Monitoring. Biosens. Bioelectron. 2006, 21, 1405-1423.

(5) Schena, M.; Shalon, D.; Davis, R. W.; Brown, P. O. Quantitative Monitoring of Gene Expression Patterns with a Complementary DNA Microarray. Science 1995, 270, 467-470.

(6) on behalf of the US National Human Genome Research Institute; Collins, F. S.; Green, E. D.; Guttmacher, A. E.; Guyer, M. S. A Vision for the Future of Genomics Research. Nature 2003, 422, $15-847$.

(7) Storhoff, J. J.; Lucas, A. D.; Garimella, V.; Bao, Y. P.; Müller, U. R. Homogeneous Detection of Unamplified Genomic DNA Sequences Based on Colorimetric Scatter of Gold Nanoparticle Probes. Nat. Biotechnol. 2004, 22, 883-887.

(8) Seeman, N. C.; Sleiman, H. F. DNA Nanotechnology. Nat. Rev. Mater. 2018, 3, 17068.

(9) Church, G. M.; Gao, Y.; Kosuri, S. Next-Generation Digital Information Storage in DNA. Science 2012, 337, 1628-1628.

(10) Maurano, M. T.; Humbert, R.; Rynes, E.; Thurman, R.; Haugen, E.; Wang, H.; Reynolds, A. P.; Sandstrom, R.; Qu, H.; Brody, J.; Shafer, A.; Neri, F.; Lee, K.; Kutyavin, T.; Stehling-Sun, S.; Johnson, A. K.; Canfield, T. K.; Giste, E.; Diegel, M.; Bates, D.; Hansen, R. S.; Neph, S.; Sabo, P. J.; Heimfeld, S.; Raubitschek, A.; Ziegler, S.; Cotsapas, C.; Sotoodehnia, N.; Glass, I.; Sunyaev, S. R.; 
Kaul, R.; Stamatoyannopoulos, J. A. Systematic Localization of Common Disease-Associated Variation in Regulatory DNA. Science 2012, 337, 1190-1195.

(11) Gaylord, B. S.; Massie, M. R.; Feinstein, S. C.; Bazan, G. C. SNP Detection Using Peptide Nucleic Acid Probes and Conjugated Polymers: Applications in Neurodegenerative Disease Identification. Proc. Natl. Acad. Sci. U. S. A. 2005, 102, 34-39.

(12) Feriotto, G.; Corradini, R.; Sforza, S.; Bianchi, N.; Mischiati, C.; Marchelli, R.; Gambari, R. Peptide Nucleic Acids and Biosensor Technology for Real-Time Detection of the Cystic Fibrosis W1282X Mutation by Surface Plasmon Resonance. Lab. Invest. 2001, 81, $1415-1427$.

(13) Das, J.; Ivanov, I.; Montermini, L.; Rak, J.; Sargent, E. H.; Kelley, S. O. An Electrochemical Clamp Assay for Direct, Rapid Analysis of Circulating Nucleic Acids in Serum. Nat. Chem. 2015, 7, 569-575.

(14) Diehl, F.; Schmidt, K.; Choti, M. A.; Romans, K.; Goodman, S.; Li, M.; Thornton, K.; Agrawal, N.; Sokoll, L.; Szabo, S. A.; Kinzler, K. W.; Vogelstein, B.; Diaz, L. A. Circulating Mutant DNA to Assess Tumor Dynamics. Nat. Med. 2008, 14, 985-990.

(15) Yong, E. Cancer Biomarkers: Written in Blood. Nature 2014, 511, 524-526.

(16) Bellassai, N.; Spoto, G. Biosensors for Liquid Biopsy: Circulating Nucleic Acids to Diagnose and Treat Cancer. Anal. Bioanal. Chem. 2016, 408, 7255-7264.

(17) Sassolas, A.; Leca-Bouvier, B. D.; Blum, L. J. DNA Biosensors and Microarrays. Chem. Rev. 2008, 108, 109-139.

(18) Wang, J. Electrochemical Nucleic Acid Biosensors. Anal. Chim. Acta 2002, 469, 63-71.

(19) Drummond, T. G.; Hill, M. G.; Barton, J. K. Electrochemical DNA Sensors. Nat. Biotechnol. 2003, 21, 1192-1199.

(20) Scrimin, P.; Prins, L. J. Sensing through Signal Amplification. Chem. Soc. Rev. 2011, 40, 4488-4505.

(21) Zanoli, L. M.; D’Agata, R.; Spoto, G. Functionalized Gold Nanoparticles for Ultrasensitive DNA Detection. Anal. Bioanal. Chem. 2012, 402, 1759-1771.

(22) Biagiotti, V.; Porchetta, A.; Desiderati, S.; Plaxco, K. W.; Palleschi, G.; Ricci, F. Probe Accessibility Effects on the Performance of Electrochemical Biosensors Employing DNA Monolayers. Anal. Bioanal. Chem. 2012, 402, 413-421.

(23) Xu, F.; Pellino, A. M.; Knoll, W. Electrostatic Repulsion and Steric Hindrance Effects of Surface Probe Density on Deoxyribonucleic Acid (DNA)/Peptide Nucleic Acid (PNA) Hybridization. Thin Solid Films 2008, 516, 8634-8639.

(24) De, A.; Souchelnytskyi, S.; Van Den Berg, A.; Carlen, E. T. Peptide Nucleic Acid (PNA)-DNA Duplexes: Comparison of Hybridization Affinity between Vertically and Horizontally Tethered PNA Probes. ACS Appl. Mater. Interfaces 2013, 5, 4607-4612.

(25) Movilli, J.; Rozzi, A.; Ricciardi, R.; Corradini, R.; Huskens, J. Control of Probe Density at DNA Biosensor Surfaces Using Poly(LLysine) with Appended Reactive Groups. Bioconjugate Chem. 2018, 29, 4110-4118.

(26) Reimhult, E.; Höök, F. Design of Surface Modifications for Nanoscale Sensor Applications. Sensors 2015, 15, 1635-1675.

(27) Yao, D.; Kim, J.; Yu, F.; Nielsen, P. E.; Sinner, E.-K.; Knoll, W. Surface Density Dependence of PCR Amplicon Hybridization on PNA/DNA Probe Layers. Biophys. J. 2005, 88, 2745-2751.

(28) Peterson, A. W.; Heaton, R. J.; Georgiadis, R. M. The Effect of Surface Probe Density on DNA Hybridization. Nucleic Acids Res. 2001, 29, 5163-5168.

(29) De Paul, S. M.; Falconnet, D.; Pasche, S.; Textor, M.; Abel, A. P.; Kauffmann, E.; Liedtke, R.; Ehrat, M. Tuned Graft Copolymers as Controlled Coatings for DNA Microarrays. Anal. Chem. 2005, 77, 5831-5838.

(30) Bizzotto, D.; Burgess, I. J.; Doneux, T.; Sagara, T.; Yu, H.-Z. Beyond Simple Cartoons: Challenges in Characterizing Electrochemical Biosensor Interfaces. ACS Sens. 2018, 3, 5-12.
(31) Schönhoff, M. Layered Polyelectrolyte Complexes: Physics of Formation and Molecular Properties. J. Phys.: Condens. Matter 2003, $15,1781-1808$.

(32) Monge, C.; Almodóvar, J.; Boudou, T.; Picart, C. SpatioTemporal Control of LbL Films for Biomedical Applications: From 2D to 3D. Adv. Healthcare Mater. 2015, 4, 811-830.

(33) Bütergerds, D.; Kateloe, C.; Cramer, C.; Schönhoff, M. Influence of the Degree of Ionization on the Growth Mechanism of Poly(Diallyldimethylammonium)/Poly(Acrylic Acid) Multilayers. J. Polym. Sci., Part B 2017, 55, 425-434.

(34) Picart, C.; Lavalle, P.; Hubert, P.; Cuisinier, F. J. G.; Decher, G.; Schaaf, P.; Voegel, J.-C. Buildup Mechanism for Poly(L-Lysine)/ Hyaluronic Acid Films onto a Solid Surface. Langmuir 2001, 17, $7414-7424$.

(35) Tezcaner, A.; Hicks, D.; Boulmedais, F.; Sahel, J.; Schaaf, P.; Voegel, J.-C.; Lavalle, P. Polyelectrolyte Multilayer Films as Substrates for Photoreceptor Cells. Biomacromolecules 2006, 7, 86-94.

(36) Tang, K.; Besseling, N. A. M. Formation of Polyelectrolyte Multilayers: Ionic Strengths and Growth Regimes. Soft Matter 2016, 12, 1032-1040.

(37) Blacklock, J.; Vetter, A.; Lankenau, A.; Oupický, D.; Möhwald, $\mathrm{H}$. Tuning the Mechanical Properties of Bioreducible Multilayer Films for Improved Cell Adhesion and Transfection Activity. Biomaterials 2010, 31, 7167-7174.

(38) Schönhoff, M.; Ball, V.; Bausch, A. R.; Dejugnat, C.; Delorme, N.; Glinel, K.; Klitzing, R. V.; Steitz, R. Hydration and Internal Properties of Polyelectrolyte Multilayers. Colloids Surf., A 2007, 303, 14-29.

(39) Klitzing, R. V. Internal Structure of Polyelectrolyte Multilayer Assemblies. Phys. Chem. Chem. Phys. 2006, 8, 5012-5033.

(40) Antipov, A. A.; Sukhorukov, G. B.; Leporatti, S.; Radtchenko, I. L.; Donath, E.; Möhwald, H. Polyelectrolyte Multilayer Capsule Permeability Control. Colloids Surf., A 2002, 198-200, 535-541.

(41) Matharu, Z.; Bandodkar, A. J.; Gupta, V.; Malhotra, B. D. Fundamentals and Application of Ordered Molecular Assemblies to Affinity Biosensing. Chem. Soc. Rev. 2012, 41, 1363-1402.

(42) Evtugyn, G. A.; Hianik, T. Layer-by-Layer Polyelectrolyte Assembles Involving DNA as a Platform for DNA Sensors. Curr. Anal. Chem. 2011, 7, 8-34.

(43) Zhou, X. C.; Huang, L. Q.; Li, S. F. Y. Microgravimetric DNA Sensor Based on Quartz Crystal Microbalance: Comparison of Oligonucleotide Immobilization Methods and the Application in Genetic Diagnosis. Biosens. Bioelectron. 2001, 16, 85-95.

(44) Caruso, F.; Rodda, E.; Furlong, D. N.; Niikura, K.; Okahata, Y. Quartz Crystal Microbalance Study of DNA Immobilization and Hybridization for Nucleic Acid Sensor Development. Anal. Chem. 1997, 69, 2043-2049.

(45) Yu, Y.; Feng, C.; Caminade, A.-M.; Majoral, J.-P.; Knoll, W. The Detection of DNA Hybridization on Phosphorus Dendrimer Multilayer Films by Surface Plasmon Field Enhanced-Fluorescence Spectroscopy. Langmuir 2009, 25, 13680-13684.

(46) Fritz, J.; Cooper, E. B.; Gaudet, S.; Sorger, P. K.; Manalis, S. R. Electronic Detection of DNA by Its Intrinsic Molecular Charge. Proc. Natl. Acad. Sci. U. S. A. 2002, 99, 14142-14146.

(47) Davila, J.; Toulemon, D.; Garnier, T.; Garnier, A.; Senger, B.; Voegel, J.-C.; Mésini, P. J.; Schaaf, P.; Boulmedais, F.; Jierry, L. Bioaffinity Sensor Based on Nanoarchitectonic Films: Control of the Specific Adsorption of Proteins through the Dual Role of an Ethylene Oxide Spacer. Langmuir 2013, 29, 7488-7498.

(48) Chen, D.; Wu, M.; Li, B.; Ren, K.; Cheng, Z.; Ji, J.; Li, Y.; Sun, J. Layer-by-Layer-Assembled Healable Antifouling Films. Adv. Mater. 2015, 27, 5882-5888.

(49) Kinnane, C. R.; Wark, K.; Such, G. K.; Johnston, A. P. R.; Caruso, F. Peptide-Functionalized, Low-Biofouling Click Multilayers for Promoting Cell Adhesion and Growth. Small 2009, 5, 444-448.

(50) Di Iorio, D.; Marti, A.; Koeman, S.; Huskens, J. Clickable PolyL-Lysine for the Formation of Biorecognition Surfaces. RSC Adv. 2019, 9, 35608-35613. 
(51) Movilli, J.; Kolkman, R. W.; Rozzi, A.; Corradini, R.; Segerink, L. I.; Huskens, J. Increasing the Sensitivity of Electrochemical DNA Detection by a Micropillar-Structured Biosensing Surface. Langmuir 2020, 36, 4272-4279.

(52) Thierry, A. R.; Mouliere, F.; El Messaoudi, S.; Mollevi, C.; Lopez-Crapez, E.; Rolet, F.; Gillet, B.; Gongora, C.; Dechelotte, P.; Robert, B.; Del Rio, M.; Lamy, P. J.; Bibeau, F.; Nouaille, M.; Loriot, V.; Jarrousse, A.-S.; Molina, F.; Mathonnet, M.; Pezet, D.; Ychou, M. Clinical Validation of the Detection of KRAS and BRAF Mutations from Circulating Tumor DNA. Nat. Med. 2014, 20, 430-435.

(53) Burke, S. E.; Barrett, C. J. pH-Responsive Properties of Multilayered Poly(L-Lysine)/Hyaluronic Acid Surfaces. Biomacromolecules 2003, 4, 1773-1783.

(54) Picart, C.; Mutterer, J.; Richert, L.; Luo, Y.; Prestwich, G. D.; Schaaf, P.; Voegel, J.-C.; Lavalle, P. Molecular Basis for the Explanation of the Exponential Growth of Polyelectrolyte Multilayers. Proc. Natl. Acad. Sci. U. S. A. 2002, 99, 12531-12535.

(55) Kuzmin, A.; Poloukhtine, A.; Wolfert, M. A.; Popik, V. V. Surface Functionalization Using Catalyst-Free Azide-Alkyne Cycloaddition. Bioconjugate Chem. 2010, 21, 2076-2085.

(56) Boulmedais, F.; Frisch, B.; Etienne, O.; Lavalle, P.; Picart, C.; Ogier, J.; Voegel, J.-C.; Schaaf, P.; Egles, C. Polyelectrolyte Multilayer Films with Pegylated Polypeptides as a New Type of Anti-Microbial Protection for Biomaterials. Biomaterials 2004, 25, 2003-2011.

(57) Schlenoff, J. B.; Rmaile, A. H.; Bucur, C. B. Hydration Contributions to Association in Polyelectrolyte Multilayers and Complexes: Visualizing Hydrophobicity. J. Am. Chem. Soc. 2008, 130, 13589-13597.

(58) Schlenoff, J. B. Zwitteration: Coating Surfaces with Zwitterionic Functionality to Reduce Nonspecific Adsorption. Langmuir 2014, 30, 9625-9636.

(59) Graf, N.; Thomasson, E.; Tanno, A.; Vörös, J.; Zambelli, T. Spontaneous Formation of a Vesicle Multilayer on Top of an Exponentially Growing Polyelectrolyte Multilayer Mediated by Diffusing Poly-L-Lysine. J. Phys. Chem. B 2011, 115, 12386-12391.

(60) Bieker, P.; Schönhoff, M. Linear and Exponential Growth Regimes of Multilayers of Weak Polyelectrolytes in Dependence on pH. Macromolecules 2010, 43, 5052-5059.

(61) Porcel, C.; Lavalle, P.; Decher, G.; Senger, B.; Voegel, J.-C.; Schaaf, P. Influence of the Polyelectrolyte Molecular Weight on Exponentially Growing Multilayer Films in the Linear Regime. Langmuir 2007, 23, 1898-1904.

(62) Bütergerds, D.; Cramer, C.; Schönhoff, M. pH-Dependent Growth Laws and Viscoelastic Parameters of Poly-L-Lysine/ Hyaluronic Acid Multilayers. Adv. Mater. Interfaces 2017, 4, 1600592.

(63) Jourdainne, L.; Arntz, Y.; Senger, B.; Debry, C.; Voegel, J.-C.; Schaaf, P.; Lavalle, P. Multiple Strata of Exponentially Growing Polyelectrolyte Multilayer Films. Macromolecules 2007, 40, 316-321.

(64) Muzzio, N. E.; Pasquale, M. A.; Gregurec, D.; Diamanti, E.; Kosutic, M.; Azzaroni, O.; Moya, S. E. Polyelectrolytes Multilayers to Modulate Cell Adhesion: A Study of the Influence of Film Composition and Polyelectrolyte Interdigitation on the Adhesion of the A549 Cell Line. Macromol. Biosci. 2016, 16, 482-495.

(65) Bertrand, P.; Jonas, A.; Laschewsky, A.; Legras, R. Ultrathin Polymer Coatings by Complexation of Polyelectrolytes at Interfaces: Suitable Materials, Structure and Properties Scheme of the Electrostatic Layer-by-Layer Self-Assembly (ESA). Macromol. Rapid Commun. 2000, 21, 319-348.

(66) Larsson, C.; Rodahl, M.; Höök, F. Characterization of DNA Immobilization and Subsequent Hybridization on a 2D Arrangement of Streptavidin on a Biotin-Modified Lipid Bilayer Supported on $\mathrm{SiO}_{2}$. Anal. Chem. 2003, 75, 5080-5087.

(67) Su, X.; Wu, Y.-J.; Knoll, W. Comparison of Surface Plasmon Resonance Spectroscopy and Quartz Crystal Microbalance Techniques for Studying DNA Assembly and Hybridization. Biosens. Bioelectron. 2005, 21, 719-726.

(68) Duan, X.; Mu, L.; Sawtelle, S. D.; Rajan, N. K.; Han, Z.; Wang, Y.; Qu, H.; Reed, M. A. Functionalized Polyelectrolytes Assembling on Nano-BioFETs for Biosensing Applications. Adv. Funct. Mater. 2015, 25, 2279-2286.

(69) Voinova, M. V.; Rodahl, M.; Jonson, M.; Kasemo, B. Viscoelastic Acoustic Response of Layered Polymer Films at FluidSolid Interfaces: Continuum Mechanics Approach. Phys. Scr. 1999, 59, 391-396.

(70) Heuberger, M.; Drobek, T.; Vörös, J. About the Role of Water in Surface-Grafted Poly(Ethylene Glycol) Layers. Langmuir 2004, 20, 9445-9448.

(71) Höök, F.; Ray, A.; Nordén, B.; Kasemo, B. Characterization of PNA and DNA Immobilization and Subsequent Hybridization with DNA Using Acoustic-Shear-Wave Attenuation Measurements. Langmuir 2001, 17, 8305-8312.

(72) Dutta, A. K.; Nayak, A.; Belfort, G. Viscoelastic Properties of Adsorbed and Cross-Linked Polypeptide and Protein Layers at a Solid-Liquid Interface. J. Colloid Interface Sci. 2008, 324, 55-60.

(73) Stengel, G.; Höök, F.; Knoll, W. Viscoelastic Modeling of Template-Directed DNA Synthesis. Anal. Chem. 2005, 77, 37093714. 